\title{
Bypass of a Liquid Taylor Drop through Successive Bends in a Rectangular Channel
}

Sudhakar Thippavathini ${ }^{1,2}$, Arup K. Das ${ }^{2 *}$

${ }^{1}$ National Institute of Technology Uttarakhand, Srinagar (Garhwal), Uttarakhand, India

${ }^{2}$ Indian Institute of Technology Roorkee, Roorkee-247667, Uttarakhand, India

*arupdas80@gmail.com 
Table S1. Model equations for multiphase lattice Boltzmann method (Sudhakar and Das (2018))

\begin{tabular}{|c|c|c|}
\hline Description & Equations & \\
\hline $\begin{array}{l}\text { Evaluation of average density based on mesoscopic } \\
\text { probability distribution function, } f_{i}\end{array}$ & $n=\frac{\rho_{L}+\rho_{H}}{2}=\sum_{i} f_{i}$ & $(\mathrm{~S} 1)$ \\
\hline $\begin{array}{l}\text { Evaluation of lattice density difference from } \\
\text { mesoscopic probability distribution function, } g_{i}\end{array}$ & $\phi=\frac{\rho_{H}-\rho_{L}}{2}=\sum_{i} g_{i}$ & $(\mathrm{~S} 2)$ \\
\hline Calculation of macroscopic velocity, $u$ & $u=\left(\sum_{i} f_{i} e_{i}^{r}+\frac{1}{2}\left(\mu_{\phi} \nabla \stackrel{r}{\phi}+\stackrel{r}{F_{b}}\right)\right) / n$ & $(\mathrm{~S} 3)$ \\
\hline Collision and propagation of $f_{i}$ 's & $f_{i}\left(\stackrel{\mathrm{r}}{\mathbf{x}}+\stackrel{\mathrm{r}}{\mathbf{e}_{i}} \delta_{t}, t+\delta_{t}\right)=\left(1-\frac{1}{\tau_{\rho}}\right) f_{i}(\stackrel{\mathrm{r}}{\mathbf{x}, t})+\frac{f_{i}^{e q}(\mathbf{r}, t)}{\tau_{\rho}}+\left(1-\frac{1}{2 \tau_{\rho}}\right) \frac{w_{i}}{c_{s}^{2}}\left(\stackrel{\mathrm{r}}{\mathbf{e}_{i}}-\stackrel{r}{u}+\frac{\underset{\mathbf{e}}{\mathrm{r}} \mathrm{r}}{c_{s}^{2}} e_{i}\right)\left(\mu_{\phi} \nabla \phi+F_{B}\right) \delta t$ & $(\mathrm{~S} 4)$ \\
\hline Equilibrium distribution of $f_{i}$ & $f_{i}^{e_{i}}(\stackrel{\mathrm{r}}{\mathbf{x}}, t)=w_{i} A_{i}+w_{i} n\left(3 e_{i \alpha} u_{\alpha}-\frac{3}{2} u^{2}+\frac{9}{2} u_{\alpha} u_{\beta} e_{i \alpha} e_{i \beta}\right)$ & (S5) \\
\hline Collision and propagation of $g_{\mathrm{i}}{ }^{\prime} \mathrm{s}$ & $g_{i}\left(\stackrel{r}{x}+\stackrel{r}{e_{i}} \Delta t, t+\Delta t\right)=g_{i}(x, t)+\frac{g_{i}^{(e q)}(\stackrel{r}{x}, t)-g_{i}(\stackrel{r}{x}, t)}{\tau_{\phi}}$ & (S6) \\
\hline Equilibrium distribution of $g_{i}$ & $g_{i}^{e q}(\bar{x}, t)=B_{i}+C_{i} \varnothing+D_{i} \varnothing \bar{e}_{i} \bar{u}$ & $(\mathrm{~S} 7)$ \\
\hline Definition of Chemical potential & $\mu_{\phi}=A\left(4 \phi^{3}-\left(\rho_{h}-\rho_{l}\right)^{2} \phi\right)-k \nabla^{2} \phi$ & $(\mathrm{S} 8)$ \\
\hline Definition of Mobility & $\theta_{M}=q\left(\tau_{\phi} q-0.5\right) \delta \Gamma$ & $(\mathrm{S} 9)$ \\
\hline
\end{tabular}

Where constants in the equilibrium distribution function $\mathrm{f}_{\mathrm{i}}$ and $\mathrm{g}_{\mathrm{i}}$ can be considered as $A_{1}=\left(\frac{9}{4} n\right)-15\left(\phi \mu_{\phi}+\frac{1}{3} n\right) / 4, A_{i=2, \ldots, 19}=3\left(\phi \mu_{\phi}+\frac{1}{3} n\right)$ and $B_{i}$

$=-3 \Gamma \mu_{\emptyset}, C_{0}=1, C_{i}=0(i \neq 0), D_{i}=1 / 2 q$, respectively. 
Initialization of macroscopic lattice information, $n$ and $\phi$ (equations S1 and S2)

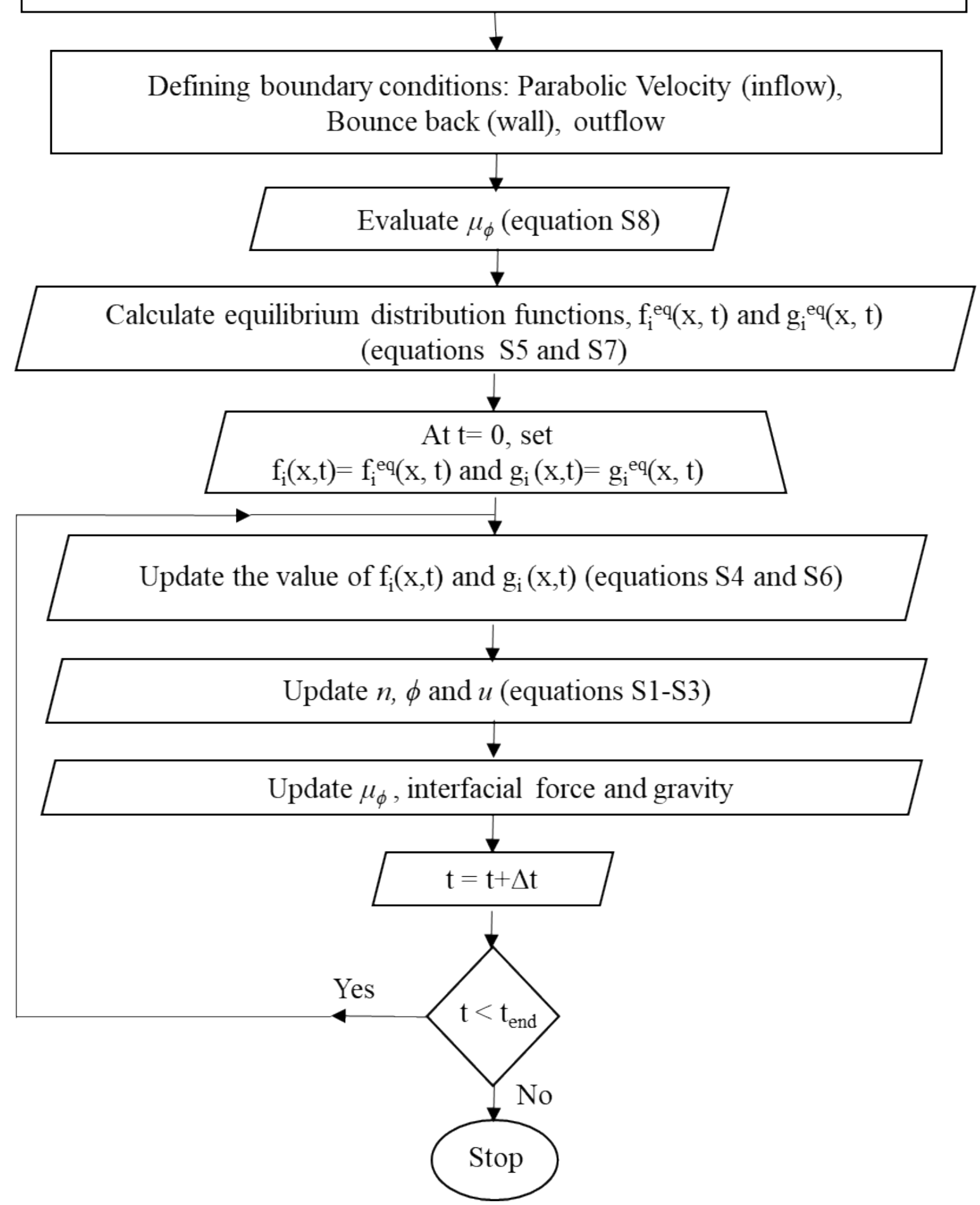

Figure S1. Multiphase lattice Boltzmann method; Solution methodology 